\title{
Daily intake of bisphenol A and potential sources of exposure: 2005-2006 National Health and Nutrition Examination Survey
}

\author{
JUDY S. LAKIND ${ }^{\mathrm{a}, \mathrm{b}, \mathrm{c}}$ AND DANIEL Q. NAIMAN ${ }^{\mathrm{d}}$

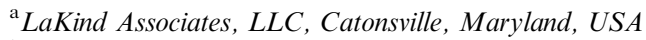 \\ ${ }^{\mathrm{b}}$ Department of Epidemiology and Preventive Medicine, University of Maryland School of Medicine, Baltimore, Maryland, USA \\ ${ }^{\mathrm{c}}$ Department of Pediatrics, Milton S. Hershey Medical Center, Penn State College of Medicine, Hershey, Pennsylvania, USA \\ ${ }^{\mathrm{d}}$ Department of Applied Mathematics and Statistics, The Johns Hopkins University, Baltimore, Maryland, USA
}

\begin{abstract}
Nationally representative data on urinary levels of bisphenol A (BPA) and its metabolites in the United States from the 2005-2006 National Health and Nutrition Examination Survey (NHANES) were used to estimate daily BPA intakes. In addition, NHANES data on potential sources of BPA exposure and personal characteristics were explored for their association with urinary BPA levels. On the basis of 2005-2006 NHANES urinary BPA data and assumptions described in this paper, median daily intake for the overall population is approximately $34 \mathrm{ng} / \mathrm{kg}$-day. Median daily BPA intakes for men are statistically significantly higher than for women; there is a significant decrease in daily BPA intake with increasing age. Gender- and age-specific median intakes differ from the overall population by less than a factor of 2 . Although estimates of daily BPA intake have decreased compared with those from the 2003-2004 NHANES, it is premature to draw conclusions regarding trends at this time, as there is no indication that BPA use declined from 2003 to 2006. On the basis of an assessment of urinary BPA and questionnaire data from the 2005-2006 NHANES, consumption of soda, school lunches, and meals prepared outside the home — but not bottled water or canned tuna — was statistically significantly associated with higher urinary BPA. Journal of Exposure Science and Environmental Epidemiology (2011) 21, 272-279; doi:10.1038/jes.2010.9; published online 17 March 2010
\end{abstract}

Keywords: bisphenol A, BPA, daily intake, United States, exposure, NHANES.

\section{Introduction}

Interest in both exposure pathways and toxicity of bisphenol A (BPA) has grown in the past few years and a major effort is underway by the US National Toxicology Program, the Food and Drug Administration, and the Centers for Disease Control and Prevention (CDC) to evaluate exposures to BPA in infants and young children, to obtain the data for constructing PBPK models, and to explore health consequences associated with perinatal exposures to BPA (Bucher, 2009). Although an important exposure pathway is thought to be from consumption of food and beverages packaged in polycarbonate bottles and epoxy-lined cans, large-scale studies on BPA levels in commonly consumed foods in the United States have not yet been published and paired data on dietary BPA intake and urinary BPA levels are needed. Information on some potential sources of BPA exposure is included in dietary questionnaire information obtained as part of the US National Health and Nutrition Examination

1. Address all correspondence to: Dr. Judy S. LaKind, Department of Epidemiology and Preventive Medicine, University of Maryland School of Medicine, LaKind Associates, LLC, 106 Oakdale Avenue, Catonsville, MD 21228, USA.

Tel./Fax: + 1410788 8639. E-mail: lakindassoc@comcast.net Received 10 December 2009; accepted 2 February 2010; published online 17 March 2010
Survey (NHANES). There are also urinary BPA data for a portion of the NHANES participants. Thus, this crosssectional database can be used to evaluate whether anticipated routes of dietary exposure are associated with urinary BPA levels. Although the NHANES database is a rich source of information on several possible BPA exposure pathways, to date, associations between potential exposure sources and urinary BPA levels from NHANES have not been explored. Various studies have examined specific products or activities for potential for human exposure to BPA, including canned foods, microwave containers (Mariscal-Arcas et al., 2009), soft drinks (Cao et al., 2009), polycarbonate bottles including baby bottles (Vandenberg et al., 2007; Carwile et al., 2009), smoking, alcohol consumption (He et al., 2009), medical procedures/products including cardiopulmonary bypass and hemodialysis (Calafat et al., 2009; FDA, 2009), dental sealants, and plastic tubing (Vandenberg et al., 2007). Personal characteristics that have been evaluated for their association with urinary BPA include age, education level, gender (He et al., 2009), household income (Becker et al., 2009), and body mass index (BMI) (Ranjit et al., 2010).

There are now two time periods for which nationally representative data on urinary BPA (CAS No. 80-05-7) levels are available for the United States: the 2003-2004 and 2005-2006 NHANES data (Calafat et al., 2008; CDC, 2009). The sample population includes males and females 
from ages 6 to $60+$ years. As excretion of ingested BPA into urine, mainly as the glucuronide conjugate, is essentially complete in $24 \mathrm{~h}$, total urinary BPA in a $24-\mathrm{h}$ urine sample approximates the BPA intake from the previous $24 \mathrm{~h}$ (LaKind and Naiman, 2008) (BPA in urine is present mainly as the glucuronide conjugate; for the purposes of this paper, the sum of the concentrations of BPA metabolites plus the parent compound in urine is referred to as "urinary BPA"). Thus, the urinary BPA data can be used to estimate daily intakes of BPA in the United States (LaKind and Naiman, 2008).

In this paper, we provide estimates of BPA daily intakes in the United States based on the 2005-2006 NHANES data and compare these with earlier published estimates from 2003 to 2004 (LaKind and Naiman, 2008). We then describe the results of an exploration of the NHANES questionnaire and examination data for statistical associations between possible sources of exposure and personal characteristics and urinary BPA levels. Of interest is whether the information available in the NHANES database supports earlier published results on likely exposure pathways. Therefore, we evaluated associations between urinary BPA levels and items from the NHANES questionnaire and examination data that specifically related to packaged food/drink consumption, smoking, and specific medical procedures. We also assessed the relationship between urinary BPA and age, gender, and $\mathrm{BMI}$ as these have been described previously.

\section{Methods}

The CDC's National Center for Health Statistics data files for NHANES 2005-2006 are available (CDC, 2009). The BPA data are from a subsample of those individuals sampled as part of NHANES $(10,348$ persons who participated in the NHANES survey during 2005 and 2006). Total BPA, after hydrolysis of conjugated metabolites, was measured in urine for NHANES 2005-2006 (analyte designated as URXBPH in the database with units of $\mathrm{ng} / \mathrm{ml}$ ). The method limit of detection (LOD) is given as $0.4 \mathrm{ng} / \mathrm{ml}$. In the data analyses, measures below the detection limit were assigned a value of the LOD divided by the square root of 2 (CDC, 2006a).
The total number of participants reported in the 20052006 subsample is 2638; however, 90 of those individuals had no data reported for BPA. Thus, the subsample for which there are existing BPA data includes 2548 individuals. Of that subsample, 182 of the measurements were below the LOD. Selected percentiles for urinary BPA for the sample population, and by gender and age, are shown in Table 1 .

\section{Estimating BPA Daily Intake}

The method used to estimate daily intake for individuals with urinary BPA data from the 2005-2006 NHANES database was described previously (LaKind and Naiman, 2008) and is summarized here. For each individual in the NHANES database with urinary BPA data, the urinary BPA concentration $(\mathrm{ng} / \mathrm{ml})$ was combined with estimated $24-\mathrm{h}$ urinary output volume $(\mathrm{ml})$ to determine daily excretion of BPA in units of ng/day. This was assumed to be equivalent to the daily intake. To obtain the daily intake adjusted for body weight (expressed in units of $\mathrm{ng} / \mathrm{kg}$-day), the daily intake for each individual was divided by that individual's body weight, as reported in the 2005-2006 NHANES database (Eq. (1)). Thirteen individuals were missing body weight data; the total count of participants with all necessary data for estimating daily BPA intake is 2535. To estimate various population (and population subgroups) quantities such as intake means or intake percentiles, weighted means and percentiles were calculated using the NHANES 2-year weights provided by CDC (as described in the NHANES analytic guidelines (CDC, 2006b)).

$$
\begin{gathered}
\text { Urinary BPA }(\mathrm{ng} / \mathrm{ml}) \times \text { urinary output }(\mathrm{ml} / \text { day }) / \\
\text { body weight }(\mathrm{kg})=\mathrm{ng} \mathrm{BPA} / \mathrm{kg} \text { day }
\end{gathered}
$$

Using this approach, distributions of intakes representative of the US population were determined for (i) all participants, (ii) participants by the following age groups: 6-11, 12-19, 20-39, 40-59, and 60+ years, and (iii) participants by gender. The calculations of point estimates and confidence intervals for the geometric mean intakes and intake percentiles were carried out in the $\mathrm{R}$ platform (Ihaka and

Table 1. Selected percentiles and geometric mean urinary BPA levels $(\mathrm{ng} / \mathrm{ml})$ from the 2005-2006 NHANES data for the sample population and by age and gender.

\begin{tabular}{lrrrrrrrr}
\hline & All & $6-11$ years & 12-19 years & $20-39$ years & 40-59 years & $60+$ years & Female & Male \\
\hline $25 \%$ & 1.0 & 1.5 & 1.3 & 1.1 & 0.7 & 0.7 & 0.8 \\
$50 \%$ & 2.0 & 2.7 & 2.4 & 2.1 & 1.7 & 1.5 & 1.8 \\
$75 \%$ & 3.7 & 5.0 & 4.3 & 3.8 & 3.1 & 3.0 & 3.7 \\
$90 \%$ & 7.0 & 13.5 & 8.4 & 7.7 & 5.9 & 6.4 & 3.7 \\
$95 \%$ & 11.5 & 22.8 & 11.9 & 11.3 & 10.0 & 8.8 & 1.7 \\
Geometric mean & 2.0 & 2.9 & 2.5 & 2.2 & 1.7 & 1.6 & 1.7 \\
$N$ & 2548 & 356 & 702 & 586 & 454 & 450 & 12.8 \\
\hline
\end{tabular}

Abbreviation: BPA, bisphenol A 
Gentleman, 1996), using the Hmisc package (http://biostat. mc.vanderbilt.edu/s/Hmisc). Confidence intervals for quantiles were calculated using a weighted bootstrap method described by LaKind et al. (2009).

As the NHANES data give information on urinary concentrations, rather than intake, certain assumptions and databases were relied on (LaKind and Naiman, 2008). First, we used urinary BPA to represent daily intake as excretion of ingested BPA (parent and metabolite) into urine is essentially complete in $24 \mathrm{~h}$ (Dekant and Völkel, 2008). Weighting factors provided by CDC were applied to the raw data, resulting in estimates of percentiles and mean values that are intended to be representative of the US population. Volumebased urinary BPA data ( $\mathrm{ng} / \mathrm{ml}$ urine) were used because the appropriateness of adjusting urinary BPA concentrations by creatinine has been questioned (Mahalingaiah et al., 2008). In our earlier analysis (LaKind and Naiman, 2008), we generally found that within age groups — with the exception of the 6-11-year olds - creatinine-adjusted intake estimates tend to be lower than intake estimates based on non-adjusted urinary BPA data and that intake estimates based on creatinine-adjusted BPA concentrations and non-adjusted concentrations are fairly highly correlated (correlation coefficients ranging from $75 \%$ to $83 \%$ ).

No 24-h urinary output data were collected for the NHANES participants; we, therefore, used generic values to describe typical urinary output based on age and gender to estimate total daily excretion of BPA in ng (Valentin, 2002; ICRP, 2003). For individuals from 20 years of age and older, "adult" ICRP values were used (male, $1600 \mathrm{ml} /$ day; female, $1200 \mathrm{ml} /$ day). For ages 12-19 years, the ICRP value for 15-year olds was used $(1200 \mathrm{ml} /$ day $)$. For ages 6-11 years, the average of the ICRP values for the 5-year olds $(500 \mathrm{ml} /$ day) and 10-year olds ( $700 \mathrm{ml} /$ day $)$ was used.

\section{Associations Between NHANES Questionnaire Data and Urinary BPA Data}

We reviewed the NHANES questionnaires to identify items that have been previously described in the published literature as possible BPA exposure pathways. We examined data from the demographics, examination, and questionnaire files located at http://www.cdc.gov/nchs/nhanes/nhanes2005-2006/ nhanes05_06.htm.

As BPA is rapidly excreted, only questions that would indicate regular BPA exposures were considered. Although the most widely discussed exposure route is from consumption of liquids stored in polycarbonate bottles, in particular exposure to bottle-fed infants, no information specific to these types of bottles was collected as part of the NHANES survey nor were children under the age of 6 years included; therefore, we could not evaluate this specific exposure route or population. We focused on questionnaire data (from the Food Frequency Questionnaire) related to diet (specifically questions that included information on consumption of canned, bottled, or packaged foods); we identified only one question in the NHANES 2005-2006 examination variable list that specifically related to consumption of canned food (tuna). We also examined questions on alcohol consumption and smoking. Alcohol as an exposure source of BPA (He et al., 2009) may be related to storage and packaging of alcohol. The basis for the reported association between smoking and BPA exposure (He et al., 2009) is unclear; it is possible that smoking is a surrogate for another behavior correlated with BPA exposure. We were also interested in items in NHANES that would provide information on individuals undergoing medical procedures that could lead to BPA exposures (Yamasaki et al., 2001). Only one question related to this potential exposure route was identified: whether the individual underwent dialysis. Finally, we examined associations between urinary BPA and age, gender, and BMI. The interested reader can link the variables used in this analysis to the questionnaire component at the following URL: http://www.cdc.gov/nchs/data/nhanes/nhanes_05_06/ varexam_d.pdf.

The predictor variables investigated for association with urinary BPA concentrations are of three types: categorical, ordinal, or numerical. We used non-parametric methods so that minimal assumptions would be made about the underlying distributions. For the ordinal and numerical predictor variables, we performed two-sided tests of association based on Spearman's rank correlation. In addition to being nonparametric, this method allows for ordinal responses in which meaningful numerical values cannot be assigned (i.e. for questions that produce ranges of values). Standard asymptotic approximations to $P$-values may be inaccurate because of the common occurrence of ties. In these instances, we used permutation tests to calculate $P$-values: for each of 100,000 trials, the ordinal data were randomly permuted and a Spearman's correlation was calculated between the permuted values and the urinary BPA values. The $P$-value is taken to be the proportion of these trials in which the obtained Spearman's correlation was at least as extreme as the one actually observed for the real data. For categorical data, we used the Wilcoxon/Mann-Whitney rank sum test to compare groups. As we are interested in associations between a predictor variable and BPA urinary concentrations and are not seeking results that are representative of the general US population, for these analyses, we did not use the CDC-weighting factors.

\section{Results}

BPA Daily Intake

Percentiles and means were estimated for BPA daily intakes from the 2005-2006 NHANES data based on individual urinary BPA data and individual body weight data as described in the previous section. Table 2 gives the results by 


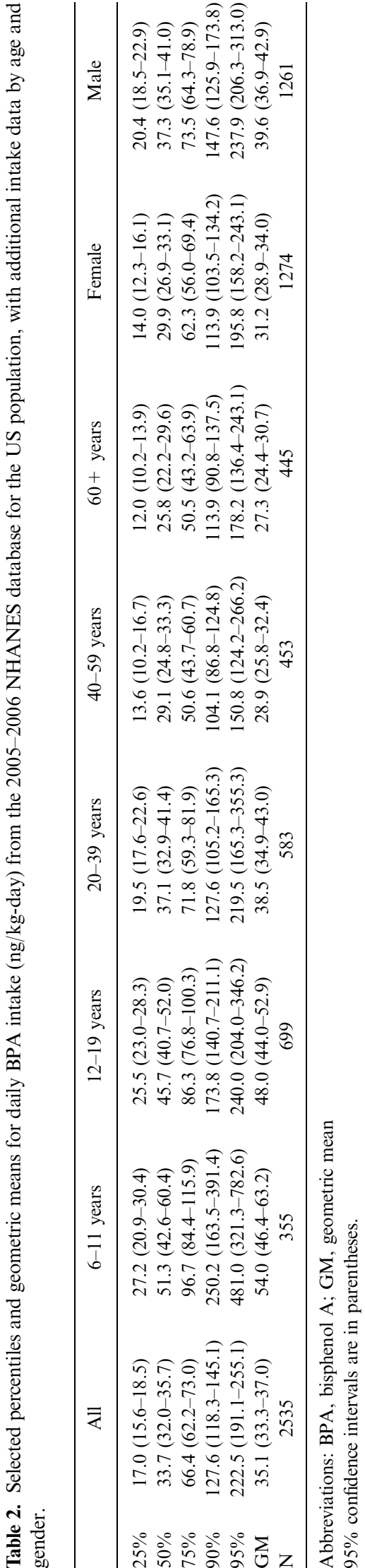

total population, age group (no data were reported by NHANES for individuals under the age of 6 years), and gender. Median BPA intakes are the highest for the 6-11-year age group, and intakes decline with increasing age $(6-11>12-19>20-39>40-59>60+)$; intakes for adults are statistically significantly less than those for children in ages 6-11 years. Median BPA intakes are statistically significantly higher for males compared with females.

\section{Associations Between Urinary BPA and Dietary Exposure and Personal/Demographic Data in NHANES}

The results of the exploration of correlations between NHANES questionnaire and examination data and urinary BPA data are described below and summarized in Table 3.

Dietary Exposure We identified several questions specifically related to consumption of canned food (tuna), bottled or canned drinks (soda or bottled water) or food that may be more likely to be packaged rather than fresh (school lunches or meals obtained from outside the home). Given the short physiologic half-life of BPA, we specifically targeted questions that would be likely to give information on recent exposures.

Soda, bottled water, canned tuna, school lunches, meals away from home There was a positive statistically significant association between urinary BPA and consumption of soda during non-summer months $(P=0.0001)$ and summer months $(P=0.0004)$, number of school lunches per week during the school year $(P=0.002)$ and meals not prepared at home $(P=0.006)$. The association between soda consumption and urinary BPA remained for those 18 years of age and older and for those younger than 18 years. For consumption of meals not prepared at home, separation of the population by age (18 years and older or $<18$ years of age) reveals that the relationship for adults remains significant $(P=0.0002)$, but for those under the age of 18 years, the relationship is no longer significant $(P=0.9)$. Consumption of canned tuna was not correlated with urinary BPA; this lack of correlation was observed for the whole population, for those younger than 18 years of age, and for those 18 and older. For consumption of bottled water, data from two successive interview days were available; bottled water consumption was inversely associated with urinary BPA only for the data from the second interview.

Personal/Demographic Information Age was negatively correlated with urinary BPA $(P<0.00001)$ and females had statistically significantly lower urinary BPA levels than males $(P=0.0002)$ (Table 1; Figure 1).

BMI was not correlated with urinary BPA $(P=0.43)$.

Alcohol and cigarettes No association was observed between alcohol consumption and urinary BPA levels. In general, smoking seems to be inversely correlated with urinary BPA. For example, individuals who reported being current smokers had lower urinary BPA levels than non-smokers $(P=0.011)$. 
Table 3. Associations between potential exposure, personal, and demographic variables in the 2005-2006 NHANES data and urinary BPA.

\begin{tabular}{|c|c|c|c|}
\hline Association to be tested & Variable ID & $N^{\mathrm{a}}$ & $P$-value $[+/-]$ \\
\hline $\operatorname{BMI}\left(\mathrm{kg} / \mathrm{m}^{2}\right)$ & BMXBMI & 2530 & 0.43 \\
\hline Age (in months) & RIDAGEMN & 2509 & $<0.00001[-]$ \\
\hline Gender $^{\mathrm{b}}$ & RIAGENDR & 2548 & 0.0002 \\
\hline Tuna consumption (number of times per day, week, month, or year) & FFQ0073 & 1700 & 0.17 \\
\hline \multicolumn{4}{|l|}{ Soda consumption (number of times per day, week, or month) ${ }^{\mathrm{c}}$} \\
\hline Non-summer months & FFQ0009B & 1621 & $0.0001[+]$ \\
\hline Summer months & FFQ0009A & 1622 & $0.0004[+]$ \\
\hline \multirow[t]{2}{*}{ Total bottled water drank yesterday (g) (two successive interview days) } & DR1BWATZ & 2434 & 0.32 \\
\hline & DR2BWATZ & 2217 & $0.012[-]$ \\
\hline School lunches (number per week during school year) & DBD381 & 857 & $\mathbf{0 . 0 0 2}[+]$ \\
\hline Meals not prepared at home (number of times per week) & DBD091 & 2524 & $0.006[+]$ \\
\hline \multicolumn{4}{|l|}{ Tobacco use } \\
\hline Current smoker? (Every day, some days, not at all) ${ }^{\mathrm{d}}$ & SMQ040 & 690 & $0.011[-]$ \\
\hline \# cigarettes per day over last 5 days? $^{\mathrm{e}}$ & SMQ720 & 391 & 0.063 \\
\hline \# cigarettes per day ${ }^{\mathrm{d}}$ & SMD070 & 279 & 0.20 \\
\hline Alcohol consumption (number drinks per week, month, or year) ${ }^{\mathrm{d}}$ & ALQ120Q & 1207 & 0.15 \\
\hline Annual household income & INDHHINC & 2410 & 0.001 \\
\hline Race/ethnicity ${ }^{\mathrm{f}}$ & RIDRETH1 & 2523 & $<0.00001$ \\
\hline
\end{tabular}

Abbreviations: BMI, body mass index; BPA, bisphenol A

Bold indicates statistically significant associations; for associations with $P<0.05,[+/-]$ denotes a positive or negative correlation.

a \# participants with both urinary BPA data and questionnaire data.

${ }^{\mathrm{b}}$ Wilcoxon test (male $>$ female).

${ }^{\mathrm{c}}$ No information was given on whether the soda was canned or bottles; it is likely that the responses represent a mixture of bottled and canned soda consumption.

${ }^{\mathrm{d}}$ Ages $20+$ years.

'encludes only those individuals who reported smoking at least one cigarette over the last 5 days.

${ }^{\mathrm{f}}$ Mexican Americans and Non-Hispanic Whites significantly lower than Non-Hispanic Blacks.

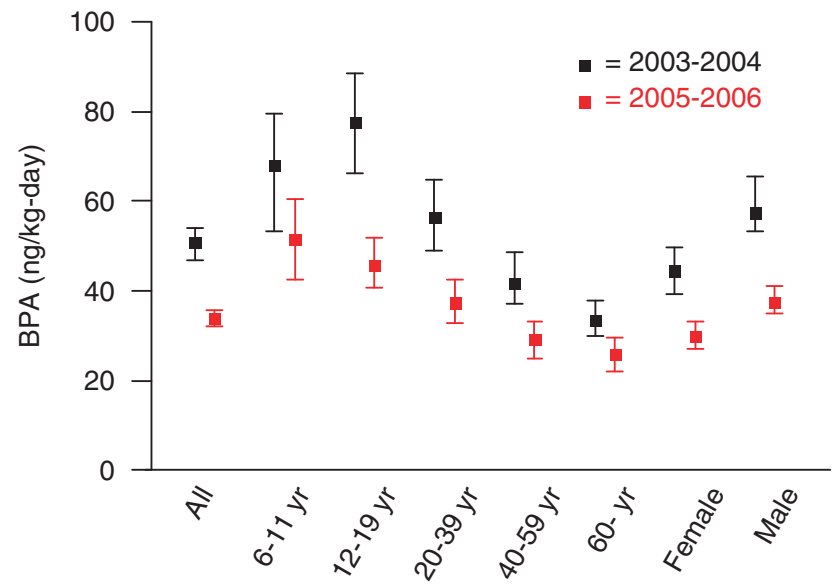

Figure 1. Median bisphenol A intakes (ng/kg-day) and 95\% CIs for the overall population and by gender and age group (2003-2004 and 2005-2006 NHANES). BPA, bisphenol A.

In addition, the number of cigarettes smoked during the earlier 5 days was negatively correlated with urinary BPA (although the association was not significant). However, we did not observe a dose-response relationship between numbers of cigarettes smoked per day and urinary BPA. Data were also available with which to evaluate the association between urinary BPA and when the participant last smoked (variable SMQ725). The highest median level was for the group of participants who last smoked the day before the questionnaire was administered $(2.7 \mathrm{ng} / \mathrm{ml})$ compared with those who smoked the day of the interview $(2.15 \mathrm{ng} / \mathrm{ml})$ and those who last smoked 3-5 days before the interview $(2.0 \mathrm{ng} / \mathrm{ml})$.

Medical procedures Only three individuals underwent dialysis over the earlier 12 months and no information was available on when the last procedure took place (although a typical dialysis patient will undergo treatment three times per week (National Kidney Foundation, 2009)). Two of the participants' urinary BPA levels were around the 75th percentiles for the general population $(3.7 \mathrm{ng} / \mathrm{ml})$ and one was below the 25 th percentile $(0.5 \mathrm{ng} / \mathrm{ml})$ (Table 1$)$.

Racelethnicity Mexican Americans had significantly lower urinary BPA levels than Non-Hispanic Blacks; Non-Hispanic Whites also had significantly lower urinary BPA levels than Non-Hispanic Blacks (Wilcoxon test, $P<0.00001$ ).

\section{Discussion}

Estimates of Daily Intake of BPA in the United States On the basis of the 2005-2006 NHANES urinary BPA data and the assumptions described in this paper, median daily 
intake for the overall population is approximately $34 \mathrm{ng} / \mathrm{kg}$ day. This is in close agreement with a recent estimate of BPA intake from Germany (30 ng/kg; Völkel et al., 2008). Median daily BPA intakes for men are statistically significantly higher than for women and there is a significant decrease in daily BPA intake with increasing age. However, gender- and age-specific median intakes (ranging from approximately 25-50 ng/kg-day) differ from the overall population intake by less than a factor of 2 . The NHANES sample population did not include children under the age of six, so no conclusions can be drawn from these data regarding daily intakes for young children. However, other studies that have reported levels of urinary BPA in children have found that the youngest children in the cohort have had the highest urinary BPA levels. For example, in a study of 3-14-year olds in Germany $(N=599)$, the 3-5-year olds had highest levels (mean $=3.55 \mu \mathrm{g} / \mathrm{l})$ (Becker et al., 2009). Similarly, Yamano et al. (2008) observed a significant decrease in urinary BPA in 94 children over a 5 -year time period from 1 st to 6 th grade. The estimate of daily geometric mean intake for children given by Becker et al. (2009) was $60 \mathrm{ng} / \mathrm{kg}$-day, comparable with our estimate of $54 \mathrm{ng} / \mathrm{kg}$-day for 6-11-year olds.

There are now two NHANES time periods with data on urinary BPA (2003-2004 and 2005-2006). Daily BPA intakes were previously reported by LaKind and Naiman (2008) for the 2003-2004 data. Figure 1 shows the comparison between daily intake of BPA from the 2003-2004 NHANES and the 2005-2006 NHANES. Although the estimated daily intakes are lower for 2005-2006, it is premature to draw any conclusions from these apparent decreases; we were unable to locate information suggesting that BPA use in the United States declined over the 2003-2006 time period and the negative public focus on BPA began after this time period. Furthermore, results must be interpreted with caution because of the limitations imposed by the underlying data and the methodology for estimating daily intake, especially those in the tails of the intake distributions because of the small numbers of individuals represented by those tails (LaKind and Naiman, 2008).

As noted earlier (LaKind and Naiman, 2008), the controversy regarding conflicting interpretation of information from studies of BPA endocrine and reproductive toxicity is recognized. Existing health-based guidance values in the United States and Europe can be compared with our estimates of daily intakes. The European Food Safety Authority (EFSA, 2007) and the US Environmental Protection Agency (EPA, 2009) give a value of $50 \mu \mathrm{g} / \mathrm{kg}$-day $(50,000 \mathrm{ng} / \mathrm{kg}$-day) as the tolerable daily intake (TDI) and reference dose (RfD), respectively. Median values were below $52 \mathrm{ng} / \mathrm{kg}$-day for all groups analyzed and the 95 th percentiles were below $500 \mathrm{ng} /$ kg-day (Table 2). Thus, median and 95th percentile intake estimates were approximately two to three orders of magnitude below the current health-based guidance value. This result is similar to that given by Ye et al. (2009) for a cohort of Norwegian women, with the estimated average daily intakes of BPA reported to be about three orders of magnitude lower than the RfD and TDI.

\section{Associations Between Urinary BPA and Diet, Lifestyle and Personal Characteristics}

Although dietary intake is thought to be the primary source of exposure to BPA (Vandenberg et al., 2007), uncertainties remain regarding relative contributions from various sources of exposure. There are many uses of BPA for which related potential exposures have not been fully assessed (e.g. digital media, electrical and electronic equipment, automobiles, sports safety equipment, electrical laminates for printed circuit boards, composites, paints, and adhesives (http://www. bisphenol-a.org/about/faq.html\#b)). Much of the focus has been on leaching of BPA from polycarbonate bottles into beverages. In our analysis of the 2005-2006 NHANES data, bottled water consumption was not associated with increased urinary BPA, but no information on bottle type (e.g. polycarbonate, polyethylene terephthalate [PET]) was given. We observed an association between increased soda consumption and urinary BPA. The questionnaire did not distinguish between consumption from cans versus bottles. Although soda bottles are generally comprised of PET and are, therefore, unlikely to be a source of BPA exposure, cans are typically coated with an epoxy resin from which BPA can migrate (Cao et al., 2009). It is likely that some portion of soda consumption reported in the NHANES study was from cans; the association between soda consumption and urinary BPA observed here is not unreasonable.

Reports of potential for BPA exposure from food packaging, including carry-out food wrapping (Vandenberg et al., 2007), cans and microwave containers (Mariscal-Arcas et al., 2009; Ye et al., 2009), and specifically canned tuna fish (Munguía-López et al., 2005), led us to focus on NHANES questions related to consumption of food likely to come into contact with these types of packaging. The only NHANES question specifically related to a canned food item was for canned tuna; however, despite the potential for migration of BPA from the lining of can coatings into canned fish (Munguía-López et al., 2005), we did not observe an association between more frequent canned tuna consumption and higher urinary BPA. We assumed that school lunches and meals prepared outside the home were more likely to include canned or microwaved foodstuffs; greater consumption of both of these meal types was associated with higher urinary BPA. We did not have information on what percentage of urine collection for school-age participants was conducted during the school year and so could not assess that part of the sample population in isolation, but the association between urinary BPA and number of school lunches consumed was still statistically significant.

We observed statistically significant inverse associations between urinary BPA and age and household income. 
In addition, men had higher urinary BPA levels than women; median levels for men and women differed by approximately $14 \%$. BMI was not correlated with urinary BPA.

In contrast with the results of He et al. (2009), we did not observe a positive correlation between urinary BPA and alcohol consumption or cigarette smoking. Insufficient information was given on types of alcohol consumed or how the alcohol was packaged to allow for a more detailed analysis. As noted earlier, the rationale for the positive correlation between cigarette smoking and BPA exposure reported by $\mathrm{He}$ et al. (2009) is unclear; the NHANES data did not yield an analogous result.

Data from cross-sectional studies such as NHANES are most appropriately used for hypothesis-generating analyses and become especially valuable when used in conjunction with other supporting information (LaKind et al., 2008). Information on associations must be interpreted with care as cross-sectional studies do not, in and of themselves, give sufficient information with which to determine causality. This is especially the case for chemicals with short physiologic half-lives and for which there are data from only one sampling event (i.e. one biological specimen per study participant). Thus, lack of an observed association between a particular potential exposure route and urinary BPA levels does not rule out such an exposure route. However, the observation that certain behaviors are associated with urinary BPA levels (e.g. consumption of school lunches and meals outside the home) suggests that these exposure pathways warrant further study. Our exploration into associations between urinary BPA and questionnaire information in some cases confirmed the results of earlier studies, and in other cases contradicted those results. Additional confirmatory research on potential exposure pathways and magnitude of exposures - including a study design that involves measurements of both urinary BPA and BPA levels in 24-h diet - is needed.

\section{Conflict of interest}

Dr. LaKind consults to both government and industry.

\section{Acknowledgements}

This work was supported by the Polycarbonate/BPA Global Group. The Polycarbonate/BPA Global Group was not involved in the design, collection, management, analysis, or interpretation of the data; or in the preparation or approval of the manuscript. The findings and conclusions in this paper represent those of the authors, and not necessarily those of the Polycarbonate/BPA Global Group.

\section{References}

Becker K., Güen T., Seiwert M., Conrad A., Pick-Fuss H., Müller J., Wittassek M., Schulz C., and Kolossa-Gehring M. GerES IV: phthalate metabolites and bisphenol A in urine of German children. Int J Hyg Environ Health 2009: 212: 685-692.

Bucher J.R. Bisphenol A: where to now? Environ Health Perspect 2009: 117: A96-A97.

Calafat A.M., Weuve J., Ye X., Jia L.T., Hu H., Ringer S., Huttner K., and Hauser R. Exposure to bisphenol A and other phenols in neonatal intensive care unit premature infants. Environ Health Perspect 2009: 117: 639-644.

Calafat A.M., Ye X., Wong L.Y., Reidy J.A., and Needham L.L. Exposure of the U.S. Population to bisphenol A and 4-tertiary-octylphenol: 2003-2004. Environ Health Perspect 2008: 116: 39-44.

Cao X.L., Corriveau J., and Popovic S. Levels of bisphenol A in canned soft drink products in Canadian markets. J Agric Food Chem 2009: 57: 1307-1311.

Carwile J.L., Luu H.T., Bassett L.S., Driscoll D.A., Yuan C., Chang J.Y., Ye X., Calafat A.M., and Michels K.B. Polycarbonate bottle use and urinary bisphenol A concentrations. Environ Health Perspect 2009: 117: 1368-1372.

CDC (Centers for Disease Control and Prevention). General Information about the NHANES 2003-2004 Laboratory Methodology and Public Data Files. January. Available at http://www.cdc.gov/nchs/data/nhanes/nhanes_03_04/ lab_c_generaldoc.pdf, accessed on 18 October $20092006 \mathrm{a}$.

CDC (Centers for Disease Control and Prevention). Analytic and Reporting Guidelines - The National Health and Nutrition Examination Survey (NHANES). National Center for Health Statistics. Available at http:// www.cdc.gov/nchs/data/nhanes/nhanes_03_04/nhanes_analytic_guidelines_ dec 2005.pdf, accessed on 18 October 2009 2006b.

CDC (Centers for Disease Control and Prevention). Documentation, Codebook, and Frequencies. Environmental Phenols and Parabens. Laboratory Survey Years: 2005-2006. Available at http://www.cdc.gov/nchs/data/nhanes/ nhanes 05 06/eph_d.pdf, accessed on 19 October 20092009.

Dekant W., and Völkel W. Human exposure to bisphenol A by biomonitoring: methods, results and assessment of environmental exposures. Toxicol Appl Pharm 2008: 228: 114-134.

EFSA (European Food Safety Authority). Opinion of the Scientific Panel on Food Additives, Flavourings, Processing Aids and Materials in Contact with Food (AFC) Related to 2,2-bis(4-hydroxyphenyl)propane. Adopted date: 29 November 2006 Available at http://www.efsa.europa.eu/EFSA/efsa_locale1178620753812_1178620772817.htm, accessed on 13 November 20092007.

EPA (United States Environmental Protection Agency). Bisphenol A, Integrated Risk Information System. Available at http://cfpub.epa.gov/ncea/iris/index. cfm?fuseaction $=$ iris.showQuickView\&substance $n$ nmbr $=0356 \#$ reforal, accessed on 13 November 20092009.

FDA (US Food and Drug Administration). Safety Assessment of BPA in Medical Products. 7 August. Available at http://www.fda.gov/downloads/ AdvisoryCommittees/CommitteesMeetingMaterials/ScienceBoardtotheFoodand DrugAdministration/UCM176835.pdf, accessed on 19 October 20092009

He Y., Miao M., Herrinton L.J., Wu C., Yuan W., Zhou Z., and Li D.K. Bisphenol A levels in blood and urine in a Chinese population and the personal factors affecting the levels. Eviron Res 2009: 109: 629-633.

ICRP. ICRP Publication 89: Basic Anatomical and Physiological Data for Use in Radiological Protection: Reference Values, Elsevier, 2003. pp. 280.

Ihaka R., and Gentleman R. R: a language for data analysis and graphics. J Comput Graph Stat 1996: 5: 299-314.

LaKind J.S., Barraj L., Tran N., and Aylward L.L. Environmental chemicals in people: challenges in interpreting biomonitoring information. $J$ Environ Health 2008: 70: 61-64.

LaKind J.S., and Naiman D.Q. Bisphenol A (BPA) daily intakes in the United States: estimates from the 2003-2004 NHANES urinary BPA data. $J$ Expo Sci Environ Epidemiol 2008: 18: 608-615.

LaKind J.S., Naiman D.Q., Hays S.M., Aylward L.L., and Blount B.C. Public health interpretation of trihalomethane blood levels in the United States: NHANES 1999-2004. J Expo Sci Environ Epidemiol 2009; e-pub ahead of print 24 June 2009.

Mahalingaiah S., Meeker J.D., Pearson K.R., Calafat A.M., Ye X., Petrozza J., and Hauser R. Temporal variability and predictors of urinary bisphenol A concentrations in men and women. Environ Health Perspect 2008: 116: 173-178.

Mariscal-Arcas M., Rivas A., Granada A., Monteagudo C., Murcia M.A., and Olea-Serrano F. Dietary exposure assessment of pregnant women to bisphenol-A from cans and microwave containers in Southern Spain. Food Chem Toxicol 2009: 47: 506-510.

Munguía-López E.M., Gerardo-Lugo S., Peralta E., Bolumen S., and SotoValdez H. Migration of bisphenol A (BPA) from can coatings into a fatty-food stimulant and tuna fish. Food Addit Contam 2005: 22: 892-898. 
National Kidney Foundation. Dialysis. Available at http://www.kidney.org/atoz/ content/dialysisinfo.cfm, accessed on 19 October 20092009.

Ranjit N., Siefert K., and Padmanabhan V. Bisphenol-A and disparities in birth outcomes: a review and directions for future research. $J$ Perinatol 2010: 30: 2-9.

Valentin J. Basic anatomical and physiological data for use in radiological protection: reference values: ICRP Publication 89. Ann ICRP 2002: 32: 5-265.

Vandenberg L.N., Hauser R., Marcus M., Olea N., and Welshons W.V. Human exposure to bisphenol A (BPA). Reprod Toxicol 2007: 24: 139-177.

Völkel W., Kiranoglu M., and Fromme H. Determination of free and total bisphenol A inhuman urine to assess daily uptake as a basis for a valid risk assessment. Toxicol Lett 2008: 179: 155-162.

Yamano Y., Miyakawa S., Iizumi K., Itoh H., Iwasaki M., Tsugane S., Kagawa J., and Nakadate T. Long-term study of urinary bisphenol A in elementary school children. Environ Health Prev Med 2008: 13: 332-337.
Yamasaki H., Nagake Y., and Makino H. Determination of bisphenol a in effluents of hemodialyzers. Nephron 2001: 88: 376-378.

Ye X., Pierik F.H., Angerer J., Meltzer H.M., Jaddoe V.W., Tiemeier H., Hoppin J.A., and Longnecker M.P. Levels of metabolites of organophosphate pesticides, phthalates, and bisphenol $\mathrm{A}$ in pooled urine specimens from pregnant women participating in the Norwegian Mother and Child Cohort Study (MoBa). Int J Hyg Environ Health 2009: 212: 481-491.

(c)

This work is licensed under the Creative Commons Attribution-NonCommercial-No Derivative Works 3.0 Unported License. To view a copy of this license, visit http:// creativecommons.org/licenses/by-nc-nd/3.0/ 